\title{
Sobrevida en cáncer de próstata de una población del centro de Colombia
}

\section{Survival in prostate cancer in a population of the center of Colombia}

\author{
Carlos Raúl Villegas, José Arnoby Chacón, Tomás Sánchez \\ - Manizales (Colombia)
}

\section{Resumen}

Antecedentes: el cáncer de próstata (CP) ocupa el primer lugar de ocurrencia en USA y en los países en desarrollo, está entre el cuarto y sexto puesto excepto en Brasil, donde es el primer cáncer en hombres. En los registros de Oncólogos del Occidente S.A. de Colombia ocupa el sexto lugar en la población general y la segunda neoplasia en el sexo masculino.

Material y métodos: se llevó a cabo un seguimiento de los pacientes tratados en Oncólogos del Occidente -Sede de Caldas- con una población de influencia de 1100000 habitantes y un total de 12000 registros analizados desde enero de 1997 hasta junio de 2012, para un informe final de 404 pacientes con cáncer de próstata.

Resultados: la media de edad fue 69 años, $73 \%$ entre 61 y 80 años; $56 \%$ en estadio clínico II (II-A: $22 \%$ y II-B:34\%) y $18 \%$ estadio IV. Escala de Gleasson menor a 6 en $37 \%$, el $33 \%$ igual a $7(4+3: 49 \%$ y $3+4: 51 \%)$. El $30 \%$ con PSA entre 4 y $10 \mathrm{ng} / \mathrm{mL}$ y $13 \%$ PSA mayor a $100 \mathrm{ng} / \mathrm{mL}$. El 36\% con algún tipo de tratamiento previo (prostatectomía:17\% y orquiectomía:9\%); $90 \%$ con Karnofsky $>80 \%$; $29 \%$ presentaron recaída y de éstos, $73 \%$ con recaída bioquímica; la radioterapia se usó en $92 \%$ (60\% dirigida al tumor primario y $16 \%$ a metástasis); el estadio T1c se encontró en $41 \%$. La sobrevida a 10 años fue $94 \%$ entre 61 y 70 años $(\mathrm{p}=0.001)$. En pacientes con Gleasson $</=7$ sobrevivió el $83 \%$, sin diferencia entre las formas $4+3$ ó $3+4(\mathrm{p}=0.9698)$. PSA $<4 \mathrm{ng} / \mathrm{mL}$ con $98 \%$ y $>20$ hasta $99 \mathrm{ng} / \mathrm{mL}$ fue $78 \%$ ( $\mathrm{p}=0.001$ ). En pacientes con recaída la sobrevida a diez años fue $68 \%$ frente a $87 \%$ en su ausencia $(\mathrm{p}<0.01)$. Media de seguimiento de 40.1 meses (rango intercuartil: 16-52). La sobrevida a cinco y diez años en etapa localizada del 92 y $79 \%$, en enfermedad metastásica fue 52 y $30 \%$ respectivamente y con enfermedad regional la sobrevida fue de $100 \%$ tanto a cinco como a diez años.

Conclusión: este trabajo analiza factores pronósticos reconocidos en la literatura mundial aplicados a pacientes con cáncer de próstata en nuestro medio, respecto a la sobrevida de cinco y diez años, como un punto de referencia que puede ayudar a los especialistas en la toma de decisiones terapéuticas y podría constituirse en una motivación para que otras instituciones colombianas generen proyectos de investigación similares. (Acta Med Colomb 2015; 40: 101-108).

Palabras clave: cáncer, próstata, factores pronósticos, supervivencia.

\section{Abstract}

Background: prostate cancer (PC) occupies the first place of occurrence in the US; in developing countries it is between the fourth and sixth place, except in Brazil where it is the first cancer in men. In the West Oncologists S.A. of Colombia records, it ranks sixth in the general population and is the second malignancy in males.

Materials and methods: a follow-up of patients treated in the West Oncologists -headquarters of Caldas- was carried out, with an influence population of 1.1 million inhabitants and a total of 12000 records analyzed from January 1997 to June 2012, for a final report of 404 patients with prostate cancer.

Results: the mean age was 69 years, $73 \%$ between 61 and 80 years; $56 \%$ in clinical stage II (II-A: $22 \%$ and II-B: $34 \%$ ) and 18\% stage IV. Gleason Scale less than 6 in 37\%, 33\% equal to 7
Dr. Carlos Raúl Villegas: Oncólogo Clínico y Radioterapeuta, Oncólogos del Occidente S.A; Dr. José Arnoby Chacón: MSc, Profesor Titular, Facultad de Ciencias para la Salud, Departamento Clínico, Universidad de Caldas; Dr. Tomás Sánchez Villegas: Internista. Manizales (Colombia).

Correspondencia. Dr. Carlos Raúl Villegas Mejía. Manizales (Colombia).

E-mail: caravim@hotmail.com Recibido: 6/V/2014 Aceptado: 23/IV/2015 
$(4+3: 49 \%$ and $3+4: 51 \%) .30 \%$ with PSA between 4 and $10 \mathrm{ng} / \mathrm{mL}$ and $13 \%$ greater than 100 ng / mL PSA. 36\% with some type of prior treatment (prostatectomy and orchiectomy 17\%: 9\%); $90 \%$ with Karnofsky $>80 \% ; 29 \%$ relapsed and $73 \%$ of those with biochemical relapse; radiotherapy was performed in $92 \%$ (60\% directed to the primary tumor and $16 \%$ to metastasis); stage T1c was found in $41 \%$. The 10 -year survival was $94 \%$ between 61 and 70 years $(p=0.001)$. In patients with Gleason $</=7,83 \%$ survived with no difference between the scores $4+3$ or $3+4$ ( $\mathrm{p}=0.9698)$. PSA $<4 \mathrm{ng} / \mathrm{ml} 98 \%$ and $>20$ to $99 \mathrm{ng} / \mathrm{mL}$ was $78 \%(\mathrm{p}=0.001)$. In patients with relapse, ten-year survival was $68 \%$ versus $87 \%$ in its absence $(\mathrm{p}<0.01)$. Mean follow-up was 40.1 months (interquartile range: 16-52). Survival at five and ten years in localized stage was $92 \%$ and $79 \%$; in metastatic disease was $52 \%$ and $30 \%$ respectively and with regional disease survival was $100 \%$ in both five and ten years.

Conclusion: this paper analyzes prognostic factors recognized in the world literature applied to patients with prostate cancer in our country, regarding the survival of five to ten years, as a point of reference that can help specialists in the therapeutic decisions and could become a motivation for other Colombian institutions to generate similar research projects. (Acta Med Colomb 2015; 40: 101-108).

Keywords: cancer, prostate, prognostic factors, survival.

\section{Introducción}

El cáncer de próstata $(\mathrm{CP})$ se constituye en un importante problema de salud a nivel mundial y actualmente se le considera la neoplasia más común en hombres (excepto el cáncer de piel) con más de 180000 nuevos casos diagnosticados anualmente y una mortalidad de 31000 hombres por año (1). En Europa el CP es la neoplasia sólida más común, con una tasa de incidencia que supera al cáncer de pulmón y al cáncer colorrectal (2).

El CP afecta más frecuentemente a los varones de mayor edad, lo que constituye una importante preocupación de salud en los países desarrollados. En estos países $15 \%$ de los cánceres en varones son $\mathrm{CP}$, en comparación con $4 \%$ de los casos de cáncer en varones en países en vías de desarrollo (3-5).

Para el año 2010 en Canadá se presenta 10\% de mortalidad, ocupando el tercer lugar con una tasa estandarizada de mortalidad (TEM) de 13.5 por 100000 habitantes. En Estados Unidos se encuentran datos de mortalidad de $10 \%$ con el tercer lugar y una TEM de 12.3 por 100000 habitantes. En México el CP ocupa el primer lugar con $16 \%$ de mortalidad y una TEM de 13.62 por 100000 habitantes. En América Latina el CP se constituye en la neoplasia más común entre los hombres después del cáncer de piel no melanoma, con una incidencia de 52350 casos para el 2010, en su mayor parte confinados a la próstata al momento del diagnóstico (69.4\%) (6). En Argentina y Brasil ocupa el segundo lugar con $13 \%$ de mortalidad y TEM de 18.63 y 17.98 respectivamente. En Colombia según el Registro Poblacional de Cáncer de Cali, se encontró una tasa de 59 por 100000 habitantes para el periodo de 2003-2007 ocupando el tercer lugar con 14\% en mortalidad de varones según datos de cáncer de las Américas en perfiles de país 2013 y una TEM de 20.30 (7).

El registro poblacional de cáncer de Manizales, Caldas, zona de influencia de Oncólogos del Occidente, reporta 423 casos de CP durante el quinquenio 2002-2006, lo que repre- senta $7.1 \%$ de 5974 casos de cáncer en el departamento de Caldas, donde ocupa el primer lugar de presencia de cáncer en hombres. En el quinquenio 2003-2007 representa 9.4\% de casos, ocupando el tercer lugar en mortalidad y el primer lugar en incidencia con 292 casos $(19.8 \%)$ con 32.6 casos por 100000 habitantes $(8,9)$.

El objetivo de este trabajo fue determinar la supervivencia de pacientes con cáncer de próstata atendidos en Oncólogos del Occidente S.A. - sede Caldas, mediante el análisis de variables de interés clínico y epidemiológico, como una primera fase de un estudio que sería extensivo para el futuro al Eje Cafetero (Quindío - Risaralda - Caldas y Norte del Valle del Cauca), donde se encuentra la población atendida por esta institución.

\section{Material y métodos}

Mediante un estudio descriptivo de seguimiento, se analizaron las historias clínicas de pacientes atendidos en Oncólogos del Occidente S.A. en la sede del Departamento de Caldas, desde enero de 1997 hasta junio de 2012 con diagnóstico histológico de $\mathrm{CP}$, enviados a valoración, estudio y tratamiento a éste centro oncológico. Las variables fueron agrupadas según las características clínicas y epidemiológicas, la intervención realizada y el resultado obtenido. Las variables cualitativas se presentan a través de proporciones y las cuantitativas con promedios y desviaciones estándar. El análisis de la supervivencia se hizo mediante el método de Kaplan-Meier y el test de log-Rank, seleccionando las categorías que mejor pudieran discriminar los grupos en riesgo. El análisis multivariado se realizó con las variables individuales que presentaran una $\mathrm{p}<0.2$.

Se utilizó la escala de Gleasson (Gleasson Score) para la clasificación internacional anatomopatológica, introducida en 1974 (10), la cual se basa en un sistema de puntuación de la arquitectura prostática que va desde 1 (Bien diferenciado) a 5 (pobremente diferenciado, definido como lesiones 
indiferenciadas con pérdida virtual completa de toda la arquitectura glandular y abandono por completo de los intentos por formar nuevas unidades glandulares). El puntaje final es el resultado de la suma de los patrones primario y secundario evaluados con un valor total entre 2 y 10 . Para el análisis se definieron grupos de riesgo, según las guías mundiales NCCN (National Comprehensive Cancer Network), clasificados en tres categorías básicas con fin pronóstico como "riesgo bajo" (Gleasson de 2 hasta 6), "riesgo medio" (Gleasson 7, tanto 4+3 como 3+4) y "riesgo alto" (Gleasson de 8 hasta 10) $(11,12)$.

Se consideró tratamiento previo positivo en aquellos pacientes que llegaron a manejo adyuvante, es decir, después de haber recibido cualquier intervención terapéutica anterior al ingreso a Oncólogos del Occidente con intento curativo, generalmente quirúrgica tipo prostatectomía radical, pero cuyo resultado histológico se presenta con factores adversos anatomopatológicos de la pieza quirúrgica que lo hacen candidato a otro tratamiento con intento curativo, basado en quimioterapia(qt), hormonoterapia (HT) o radioterapia (RT). El tratamiento previo se presentó en $25 \%$ de la población estudiada.

En el caso del antígeno prostático específico (PSA, por su sigla en inglés) se tomó la cifra inicial anotada en la historia clínica en $\mathrm{ng} / \mathrm{mL}$ y previa a todo tipo de manipulación terapéutica paliativa o curativa. El punto de corte de riesgo se tomó con base en el resultado de estudios de tamizaje correspondiendo a $4.0 \mathrm{ng} / \mathrm{mL}(13-15)$ y con base en este tópico se conformaron subgrupos así: PSA menor de $4 \mathrm{ng} / \mathrm{mL}$, de 4-10 ng/mL, de $11-20 \mathrm{ng} / \mathrm{mL}$, de $21-99 \mathrm{ng} / \mathrm{mL}$ y mayor o igual a $100 \mathrm{ng} / \mathrm{mL}(16)$.

El estado en el último control de los pacientes se clasificó según lo registrado en la última nota médica, definido como VSAT (paciente vivo sin actividad tumoral por clínica o por laboratorio), VCAT (paciente vivo con evidencia clínica o paraclínica de enfermedad), PCAT y PSAT (pacientes perdidos con y sin actividad tumoral). Esta información debía ser anterior a un año del cierre del estudio en junio de 2012. Los datos fueron validados por diferentes medios (vía telefónica, correo, visitas domiciliarias, etc.). Los fallecidos se clasificaron en dos subgrupos, MCAT y MSAT (Muerto con o sin actividad tumoral respectivamente), según la última nota médica del control más cercano a la fecha del deceso.

El estadiaje inicial del paciente se basó en la Clasificación de la American Joint Committee on Cáncer - AJCC Cancer Staging Manual Seventh Edition (2010), con datos extractados de la nota médica inicial, del informe de la biopsia o del informe de anatomía patológica de la cirugía radical. Estos datos se homologaron y actualizaron según la AJCC $7^{\text {a }}$ Edición, en la cual se combina la información del estado del Tumor (T), los nódulos linfáticos $(\mathrm{N})$ y la presencia o ausencia de metástasis a distancia $(\mathrm{M})$, sumado a la información del Gleasson en el tumor primario y el nivel de PSA para la clasificación adecuada del riesgo de recurrencia. Luego se realizó la clasificación del riesgo según guías actualizadas
NCCN-2014 conformando grupos de bajo, medio y alto riesgo (12); igualmente se acogió la clasificación descrita en el programa SEER, definido como enfermedad localizada, intermedia y metastásica como otra variable de análisis final comparativo $(4,5)$.

Las variables se tomaron con el mayor nivel de medición anotado en la historia clínica y el agrupamiento por categorías se hizo en la etapa de análisis de resultados. Las categorías establecidas para las variables cuantitativas se basaron en puntos de corte obtenidos de la revisión de la literatura.

El proyecto fue avalado por el Comité de Ética de la Universidad de Caldas, la Dirección Médica y el Comité de Investigaciones de Oncólogos del Occidente y se permitió la aparición de su razón social en los textos que tengan como fin la publicación de resultados. Para el análisis de la información se utilizaron los programas EpiInfo 6.04d EpiInfo $^{\text {TM }}$ Versión3 .5.4y SPSS versión 14.5.

Dado que no hay intervención terapéutica directa con los pacientes ni información externa particular de los mismos, se considera una investigación sin riesgo según el artículo 11 de la Resolución № 008430 de 1993 del Ministerio de Salud de Colombia.

\section{Resultados}

Mediante un estudio descriptivo de seguimiento se analizaron 404 casos de pacientes con cáncer de próstata, con las características descritas en la Tabla 1.

Los pacientes recibieron intervenciones como se describe en la Tabla 2.

Los resultados de las intervenciones se describen en la Tabla 3, en la cual se determina la presencia de recaídas y el estado final al término de seguimiento a junio de 2012.

El seguimiento se determinó como el periodo en meses transcurrido entre el final del tratamiento y la fecha del último control o la fecha de cierre del estudio, para lo cual se establecieron los diferentes niveles de supervivencia según la Tabla 4.

\section{Discusión}

Se analizó la supervivencia a 5 y 10 años en 404 pacientes que reunieron los criterios de inclusión con base en las diferentes variables de interés general, como se describen a continuación.

\section{Características de los pacientes}

Al momento del diagnóstico el $73 \%$ de los pacientes tenían entre 61-80 años de edad con una media de 69 años $(\mathrm{DE}=8.6)$. Al igual que en otros estudios, se considera la edad como un factor muy importante asociado al desarrollo del cáncer de próstata y, como es referido por “De Vita et al, no hay otro tumor en adultos que esté más relacionado con la mayor edad que el cáncer de próstata" (17).

Los datos generales muestran una tendencia al aumento de frecuencia de presentación con el avance de la edad, pues el diagnóstico clínico difícilmente se hace en menores de 40 
Tabla 1. Características generales de los pacientes. $(n=404)$.

\begin{tabular}{|c|c|c|}
\hline Característica & $\mathbf{N}$ & $\%$ \\
\hline Edad & Media: $69 \mathrm{D}$ & \\
\hline \multicolumn{3}{|l|}{ Grupos de edad } \\
\hline Menor o igual de 60 años & 67 & 16.6 \\
\hline 61 a 70 & 151 & 37.4 \\
\hline 71 a 80 & 145 & 35.9 \\
\hline 80 y más & 41 & 10.1 \\
\hline PSA (ng/mL) & Media: $41.4 \mathrm{I}$ & \\
\hline \multicolumn{3}{|l|}{ Grupo de PSA (ng/mL) } \\
\hline Menor ó igual a 4 & 32 & 7.9 \\
\hline $5-10$ & 121 & 30.0 \\
\hline 11 a 20 & 88 & 21.8 \\
\hline 21 a 99 & 78 & 19.3 \\
\hline Igual o mayor a 100 & 51 & 12.6 \\
\hline NR & 34 & 8.4 \\
\hline \multicolumn{3}{|l|}{ Etapa clínica } \\
\hline I & 71 & 17.6 \\
\hline II & 227 & 56.2 \\
\hline III & 34 & 8.4 \\
\hline IV & 72 & 17.8 \\
\hline \multicolumn{3}{|l|}{ ETAPA } \\
\hline Localizada & 298 & 73.8 \\
\hline Metástasis & 72 & 17.8 \\
\hline Regional & 34 & 8.4 \\
\hline \multicolumn{3}{|l|}{ TIPO } \\
\hline I & 71 & 17.6 \\
\hline IIA & 89 & 22.0 \\
\hline IIB & 138 & 34.2 \\
\hline III & 34 & 8.4 \\
\hline IV & 72 & 17.8 \\
\hline \multicolumn{3}{|l|}{ Escala de Gleason } \\
\hline Menor o igual a 6 & 147 & 36.4 \\
\hline 7 & 132 & 32.7 \\
\hline $8-10$ & 94 & 23.3 \\
\hline Sin registro & 31 & 7.7 \\
\hline \multicolumn{3}{|c|}{ Tratamiento previo (Curativo o Paliativo) } \\
\hline SI & 145 & 35.9 \\
\hline NO & 259 & 64.1 \\
\hline \multicolumn{3}{|l|}{ Karnofsky inicial } \\
\hline $50-70 \%$ & 39 & 9.7 \\
\hline Más de $80 \%$ & 365 & 90.3 \\
\hline \multicolumn{3}{|l|}{ TUMOR } \\
\hline $\mathrm{T} 1$ & 189 & 46.8 \\
\hline $\mathrm{T} 2$ & 110 & 27.2 \\
\hline $\mathrm{T} 3$ & 33 & 8.2 \\
\hline $\mathrm{T} 4$ & 71 & 17.6 \\
\hline Sin clasificar & 1 & 0.2 \\
\hline \multicolumn{3}{|l|}{$\mathrm{T} 1 \mathrm{c}$} \\
\hline Otro & 244 & 60.4 \\
\hline $\mathrm{T} 1 \mathrm{C}$ & 160 & 39.6 \\
\hline
\end{tabular}

Tabla 2. Intervenciones realizadas $(n=404)$.

\begin{tabular}{|l|cc|}
\hline \multicolumn{2}{|c|}{ Intervención } \\
\hline Cirugía & 334 & 82.7 \\
Otra & 70 & 17.3 \\
Prostatectomia radical & & \\
\hline Radioterapia & 163 & 40.3 \\
Otra & 241 & 59.7 \\
Al primario & & \\
\hline
\end{tabular}

Tabla 3. Resultados de la intervención ( $n=404)$.

\begin{tabular}{|c|c|c|}
\hline \multicolumn{3}{|c|}{ Resultado } \\
\hline Seguimiento (meses) & Media: 40.0 & 34.2 \\
\hline \multicolumn{3}{|l|}{ Recaída } \\
\hline Sí & 118 & 29.2 \\
\hline No & 252 & 62.4 \\
\hline Persistencia & 34 & 8.4 \\
\hline Tiempo de recaída (Meses) & Media: 35.8 & 29.4 \\
\hline \multicolumn{3}{|l|}{ Recaída } \\
\hline Sí & 118 & 29.2 \\
\hline No & 286 & 70.8 \\
\hline \multicolumn{3}{|l|}{ Recaída bioquímica } \\
\hline Sí & 86 & 72.8 \\
\hline No & 32 & 27.2 \\
\hline \multicolumn{3}{|l|}{ Estado último control } \\
\hline Muerto con actividad tumoral (MCAT) & 40 & 9.9 \\
\hline Muerto sin actividad tumoral (MSAT) & 6 & 1.5 \\
\hline Perdido con actividad tumoral (PCAT) & 21 & 5.2 \\
\hline Perdido sin actividad tumoral (PSAT) & 16 & 4.0 \\
\hline Vivo con actividad tumoral (VCAT) & 42 & 10.4 \\
\hline Vivo sin actividad tumoral (VSAT) & 279 & 69.1 \\
\hline \multicolumn{3}{|l|}{ Resultado } \\
\hline Vivo & 364 & 90.1 \\
\hline Muerto en relación con el tumor & 40 & 9.9 \\
\hline
\end{tabular}

años, pero la incidencia aumenta rápidamente a partir de este momento y, según datos del National Cancer Institute's Surveillance, Epidemiology and End Results Program (SEER Program), los casos nuevos de CP fueron de $0.1 \%$ entre 50 y 60 años, $0.6 \%$ entre 61 y 70 años y $1 \%$ en hombres de más de 70 años $(18,19)$.

$\mathrm{Al}$ igual que en otros tumores, en pacientes mayores de 80 años parece disminuir la frecuencia, pero esta tendencia puede ser explicada por mayor comorbilidades y una disminución en la práctica de biopsias en este grupo etario, como es reportado en SEER / USA-NCI en hombres del 2000-2006 $(4,20)$. Otra posible causa de subdiagnóstico o 
Tabla 4. Supervivencia a 5 y 10 años según variables.

\begin{tabular}{|c|c|c|c|}
\hline \multirow[t]{2}{*}{ Variable } & \multicolumn{2}{|c|}{ Supervivencia a } & \multirow[t]{2}{*}{$\mathbf{p}$} \\
\hline & 5 años (\%) & 10 años (\%) & \\
\hline \multicolumn{4}{|l|}{ Edad } \\
\hline$<60$ & 83 & 83 & 0.001 \\
\hline $61-70$ & 97 & 94 & \\
\hline $71-80$ & 83 & 67 & \\
\hline$>80$ & 60 & 33 & \\
\hline \multicolumn{4}{|l|}{ Subtipo } \\
\hline T1c (localizado) & 93 & 75 & 0.02 \\
\hline \multicolumn{4}{|l|}{ Etapa clínica } \\
\hline I & 93 & 82 & 0.001 \\
\hline II & 90 & 77 & \\
\hline III & 100 & 100 & \\
\hline IV & 52 & 30 & \\
\hline \multicolumn{4}{|l|}{ Gleasson } \\
\hline$<6$ & 95 & 83 & 0.001 \\
\hline 7 & 91 & 83 & \\
\hline $8-10$ & 69 & 61 & \\
\hline No Reg. & 58 & 29 & \\
\hline \multicolumn{4}{|l|}{ Gleasson 7} \\
\hline$(4+3)$ & 92 & 82 & 0.9698 \\
\hline$(3+4)$ & 90 & 84 & \\
\hline \multicolumn{4}{|l|}{ PSA } \\
\hline$<=4$ & 98 & 98 & 0.001 \\
\hline $5-10$ & 98 & 89 & \\
\hline $11-20$ & 99 & 88 & \\
\hline $21-99$ & 86 & 78 & \\
\hline$=/>100$ & 36 & 25 & \\
\hline No reg. & 80 & 51 & \\
\hline \multicolumn{4}{|l|}{ Karnofsky inicial } \\
\hline$>80$ & 89 & 78 & 0.001 \\
\hline $50-70$ & 49 & 49 & \\
\hline \multicolumn{4}{|l|}{ Tratamiento previo } \\
\hline Sí & 84 & 67 & 0.5553 \\
\hline No & 86 & 82 & \\
\hline \multicolumn{4}{|l|}{ Tipo de cirugía } \\
\hline Prostatectomia radical & 95 & 95 & 0.001 \\
\hline Otras & 82 & 61 & \\
\hline \multicolumn{4}{|l|}{ Recaída } \\
\hline Sí & 80 & 68 & 0.0145 \\
\hline No & 90 & 87 & \\
\hline \multicolumn{4}{|l|}{ Radioterapia } \\
\hline Próstata & 94 & 85 & 0.001 \\
\hline Otras & 75 & 58 & \\
\hline \multicolumn{4}{|l|}{ Estado general } \\
\hline Localizado & 92 & 79 & 0.001 \\
\hline Regional & 100 & 100 & \\
\hline Metastásico & 52 & 30 & \\
\hline
\end{tabular}

subregistro en mayores de 80 años lo constituye el hecho de que el CP se ha considerado una enfermedad de viejos con ocurrencia de enfermedad crónica (21). Sumado a lo anterior está el hecho de que muchos más casos no llegan a ser clínicamente evidentes, como se indicó en series de autopsias, donde el CP es detectado en menores de 80 años en $30 \%$ y en hombres mayores a 80 años en $60 \%$, lo que define su crecimiento lento que lleva a muchos hombres a morir de otras causas antes de su detección (22).

Un estudio realizado por Sakr et al. en grupos de hombres blancos, negros, latinos (colombianos) y japoneses viviendo en Japón, muertos por causa diferente a cáncer de próstata, a los cuales se les examinó la próstata y los resultados se relacionaron según grupos de edad, encontrándose una mayor frecuencia de lesiones neoplásicas a medida que los hombres tenían mayor edad, con resultados que van desde $2,29,32,55$ y $64 \%$ según pertenecieran a la tercera, cuarta, quinta, sexta y octava década de la vida, respectivamente (23). En la Figura 1 se muestran los resultados de nuestro estudio en relación con la mortalidad por grupos etáreos. Se encontró sobrevida mayor en el subgrupo de 61-70 años, la cual comienza a disminuir a medida que aumenta la edad, explicado posiblemente por comorbilidades comunes a este grupo poblacional, caracterizándose así como una enfermedad crónica por el desarrollo lento de la neoplasia con sobrevidas a cinco años de $97 \%$ en el grupo de 61-70 años y de $60 \%$ para mayores de 80 años.

La etapa clínica al diagnóstico fue clasificada como I $(17.6 \%)$, II $(56.2 \%)$, III $(8.4 \%)$ y IV $(17.8 \%)$, con resultados en supervivencia similar al estudio de Braun DP et al. donde analizó 767 pacientes, encontrando una distribución por etapa clínica I (1\%); II (61\%); III (10\%); IV (14\%) y $3 \%$ de NO determinados (24).

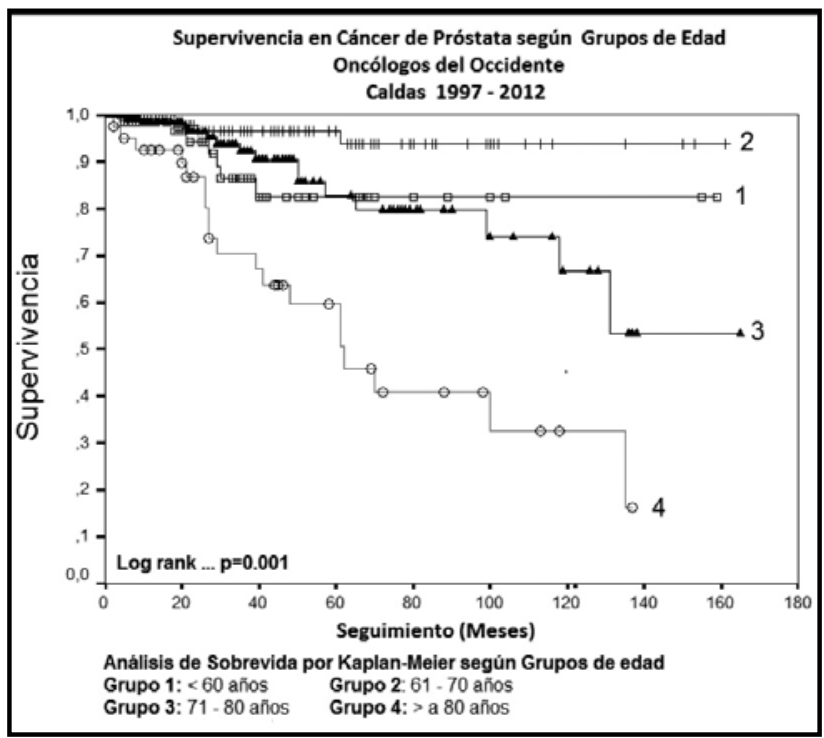

Figura 1. Análisis de sobrevida por Kaplan-Meier según Grupos de Edad. Oncólogos del Occidente S.A. - Caldas 1997-2012. Grupo 1: <60 años; Grupo 2: 61 - 70 años; Grupo 3: 71 - 80 años; Grupo 4: $>80$ años. 


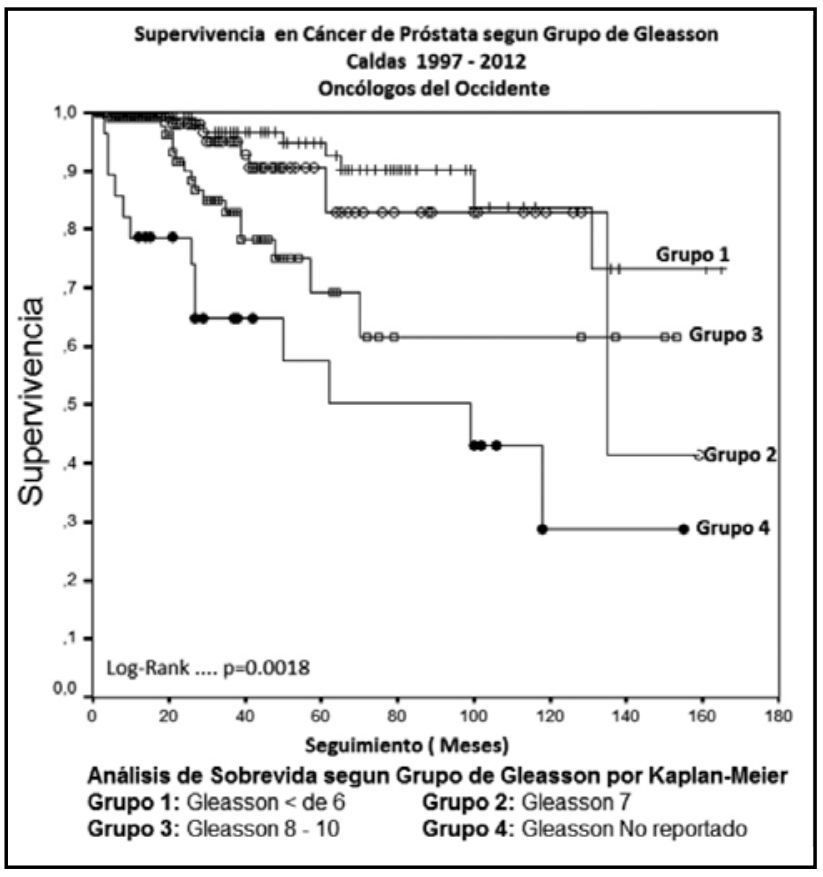

Figura 2. Análisis de Sobrevida por Kaplan-Meier según Gleasson Score. Oncólogos del Occidente SA - Caldas 1997-2012. Grupo 1: Gleasson 2 a 6; Grupo 2: Gleasson 7; Grupo 3: Gleasson 8 a 10; Grupo 4: No Reportado.

La mayor presentación en etapas localizadas es explicada en parte por el empleo rutinario de PSA como método de detección de pacientes y a quienes se les indica biopsia temprana. El hallazgo de PSA elevado conlleva a la toma de biopsia, la cual, si es positiva, clasifica el caso como un estadio T1c. La sobrevida para etapas II y III (90 y $100 \%$ a cinco años y, 77 y $100 \%$ a 10 años) frente a la etapa IV, cuya supervivencia se encontró de $52 \%$ a cinco años y $30 \%$ a 10 años, lo cual es similar a la serie del Hospital de Cáncer de Río de Janeiro I (INCA-RJ), de 1990 a 1994 con una sobrevida general a cinco años de $80 \%$ en pacientes con cáncer de próstata localizado (estadios I-II) y cerca de $40 \%$ en pacientes con metástasis a distancia (estadio IV) (25) (Tabla 4 y Figura 2).

Se encontró enfermedad metastásica en $18 \%$ en nuestro estudio frente a $4 \%$ en la serie SEER. La agrupación según la extensión de la enfermedad (localizada, regional o metastásica) muestra sobrevida muy similar a otros estudios así: en enfermedad localizada de $100 \%$ a cinco años comparada con $31.9 \%$ en enfermedad metastásica. Al igual que en otras publicaciones la enfermedad metastásica a nivel óseo constituyó la presentación predominante $(4,5)$.

Según la clasificación de Gleasson y siguiendo la recomendación dada por el College of American Pathologists de agrupar en tres grupos (2-6, 7, 8-10) definidos en Bostwick 2000, el primer grupo se presentó en $36.4 \%$ de los casos con supervivencia a cinco años de 95 y $83 \%$ a 10 años. En el grupo de Gleasson de 8-10, se obtuvo sobrevida a cinco años de $69 \%$ y a 10 años de $61 \%,(p=0.0018)$, lo que refleja más

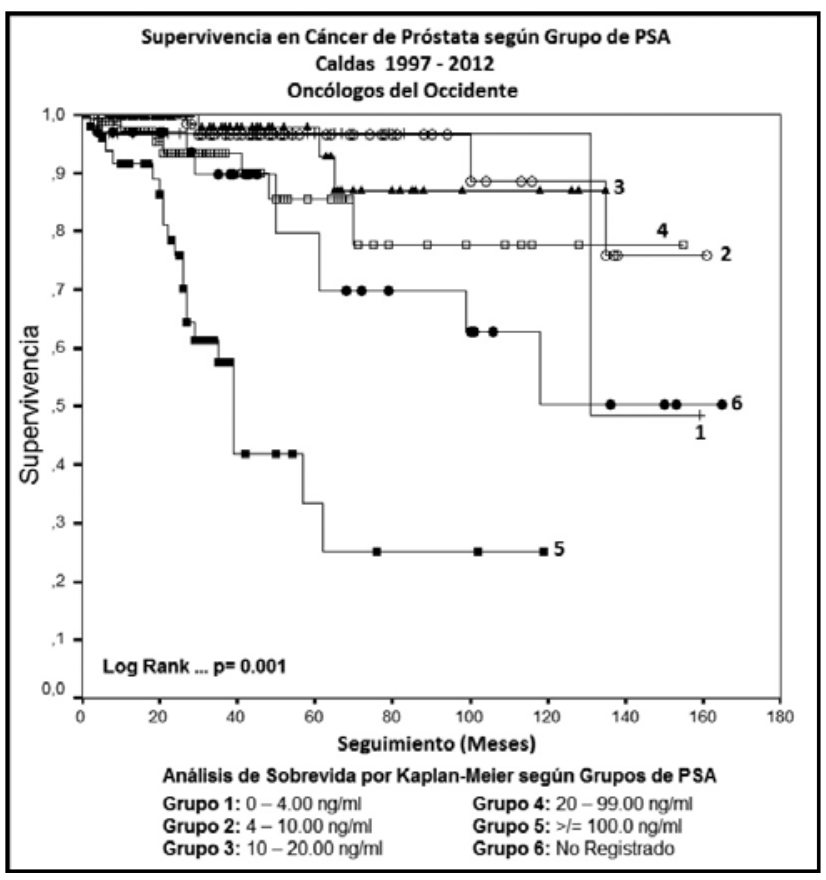

Figura 3. Análisis de Sobrevida por Kaplan-Meier según Grupos de PSA. Oncólogos del Occidente SA - Caldas 1997-2012. Grupo 1: $0-4.00 \mathrm{ng} / \mathrm{mL} ;$ Grupo 2: $4-10.00 \mathrm{ng} / \mathrm{mL}$; Grupo 3: 10 - $20.00 \mathrm{ng} / \mathrm{mL}$; Grupo 4: $20-99.00 \mathrm{ng} / \mathrm{mL}$; Grupo 5: >/= $100.0 \mathrm{ng} / \mathrm{mL}$; Grupo 6: No Registrado.

agresividad a medida que hay mayor indiferenciación de las células prostáticas, similar a otros datos reportados $(26,27)$.

El subgrupo Gleasson 7 se presentó en $33 \%$ de los casos con una sobrevida de $91 \%$ a cinco años y $83 \%$ a 10 años $(\mathrm{p}=0.0018)$, similar a la frecuencia de presentación en otros grupos y definido como grupo de buen pronóstico, pero con un patrón diferente, según los subgrupos que lo conforman. Esta distribución ha ido variando en el tiempo y en la era del tamizaje con PSA de aplicación rutinaria, por lo que más hombres son ahora diagnosticados con Gleasson 7 (28-30, 38). En el análisis por subgrupos (4+3) o (3+4)obtuvimos un resultado diferente a otros estudios, pues el subgrupo de Gleasson $7(4+3)$ fue $49 \%$ y Gleasson $7(3+4)$ de $51 \%$ con una sobrevida a cinco años de 92 y $90 \%$ y a 10 años de 82 y $84 \%(p=0.9698)$ respectivamente, contrario a lo reportado por otros autores, que indican pronóstico desfavorable en el grupo de $4+3$, con una mortalidad específica tres veces mayor en el subgrupo de 3+4 (28-30) (Figura 2). Nuestros resultados son similares al estudio de Marbele $\mathrm{S}$ et al. realizado en una población de 200 pacientes por el Departamento de Patología de Campinas, Brasil en 2007, en el cual no se encontró una diferencia significativa en cuanto a sobrevida y tiempo libre de progresión (38).

Según el PSA la sobrevida a cinco y 10 años en el grupo menor a $4 \mathrm{ng} / \mathrm{mL}$ (8\%) fue de $98 \%$, tanto a cinco como a 10 años, la cual va disminuyendo a medida que aumenta el nivel de PSA. Este hecho coincide con lo reportado por otros autores como un factor pronóstico importante, puesto que para el grupo mayor de $100 \mathrm{ng} / \mathrm{mL}$ (13\%) la sobrevida 
es $36 \%$ a los cinco años y $25 \%$ a los 10 años $(\mathrm{p}=0.001)(6$, 31, 32) (Figura 3).

Al ingreso a Oncólogos del Occidente los pacientes habían recibido tratamiento quirúrgico previo en $36 \%$ (prostatectomía radical, orquiectomía y/o linfadenectomía) cuya descripción quirúrgica correspondía a un procedimiento radical. La sobrevida a 10 años fue de $67 \%$ en pacientes con tratamiento previo y de $82 \%$ en quienes no lo habían recibido $(\mathrm{p}=0.5553)$. Esta diferencia puede ser explicada por la presencia de otros factores en los pacientes previamente tratados, tales como el puntaje de Gleasson, las cifras de PSA previas, el grado histológico o por la recaída bioquímica o clínica, razones que posiblemente indicaron el tratamiento de rescate.

El estado funcional inicial de los pacientes establecido por Karnofsky mayor a $80 \%$, fue de $90 \%$ con una sobrevida a cinco años de $89 \%$ y a 10 años de $78 \%$, comparado con el grupo de Karnofsky entre 50 y $70 \%$, cuya sobrevida fue de $49 \%$ a cinco años. Esto coincide con lo reportado por De Vita, donde se aprecia que el estado funcional es factor pronóstico, no sólo en cáncer de próstata sino en todo tipo de neoplasias, siendo usado como indicador de sobrevida, predictor de respuesta terapéutica y decisor del tipo de tratamiento (12).

Se presentó recaída en $29.2 \%$ de los casos (72.8\% de tipo bioquímico y $8.4 \%$ con enfermedad metastásica o persistencia del estado metastásico inicial), con una sobrevida a cinco años de 80 y $68 \%$ a 10 años; los que no presentaron recaída tuvieron sobrevida a cinco años de 90 y $87 \%$ a 10 años ( $\mathrm{p}=0.01)$, lo que concuerda con otras publicaciones en las cuales la presencia de recaída disminuye considerablemente el pronóstico de los pacientes, similar a los resultados obtenidos en un estudio por Leborgne en Uruguay en el 2006 de $90 \%$ a cinco años $(33,34)$.

El $92.2 \%$ de los pacientes recibió radioterapia, de los cuales $59.7 \%$ como tratamiento inicial radical único dirigido a la próstata con intento curativo, $15.1 \%$ con intención paliativa por enfermedad metastásica y $8.4 \%$ por recaída bioquímica, mientras que $9 \%$ de ellos recibió radioterapia como tratamiento adyuvante a raíz del informe patológico posquirúrgico (borde positivo, cápsula positiva y vesículas positivas). La sobrevida a cinco años fue de $94 \%$ y a 10 años de $85 \%$, cuando el tratamiento se dirigió inicialmente a la próstata con intención curativa inicial, frente a una sobrevida de $75 \%$ a cinco años y de $58 \%$ a los 10 años, cuando la terapia se empleó por las otras razones mencionadas $(\mathrm{p}=0.001)(35)$.

En nuestra serie el $41 \%$ de los pacientes fueron clasificados como T1c, con una sobrevida de $93 \%$ a cinco años y $75 \%$ a los 10 años, frente a 79 y $68 \%$ respectivamente, en el grupo clasificado como enfermedad localizada diferente a T1c ( $\mathrm{p}=0.0202)$. Este resultado concuerda con un reporte de sobrevida a ocho años del $86 \%$, hecho por Hung para este grupo clínico, pero quizás con un patrón diferente según sean evaluadas otras variables (Gleasson, PSA y T del TNM), lo cual se constituye en objeto de una futura publicación (36).

\section{Características de la intervención}

El 25\% de los pacientes recibió manejo quirúrgico tipo cirugía radical (los cuales llegaron a tratamiento adyuvante por factores adversos anatomopatológicos de la pieza quirúrgica) o habían sido tratados con orquiectomía, linfadenectomía y procedimientos mixtos por el grupo de urología.

Se aplicó radioterapia como tratamiento primario al $59.7 \%$, representado básicamente por el grupo de pacientes que no fueron candidatos idóneos para cirugía, según decisión de una junta médica urológica. Los principales factores considerados en la toma de decisión no quirúrgica fueron, entre otros: comorbilidades de índole cardiopulmonar, estado metabólico, historia quirúrgica personal, nivel de Gleasson de riesgo intermedio o alto, nivel inicial de PSA elevado, estatificación inicial elevada o por decisión propia del paciente de rechazar la intervención quirúrgica. El 40.3\% de los pacientes recibieron radioterapia para tratamiento de metástasis, recaída bioquímica, condiciones mixtas o causas posquirúrgicas como bordes quirúrgicos positivos, cápsula positiva o compromiso de vesículas seminales.

\section{Resultado de la intervención}

El seguimiento global de los pacientes fue de 40 meses en promedio (DE: 34.2 meses) con un mínimo de seis meses y un máximo de 165 meses, lo cual permitió una observación de desenlaces muy importantes, similar a lo descrito en el estudio de Migowski en Río de Janeiro (6).

La enfermedad regional o aquella definida como estadios clínicos II y III, se presentó en 12\% en SEER 2002-2008 frente a $8 \%$ en Oncólogos del Occidente con una sobrevida a cinco años de $100 \%$ en ambos trabajos, lo que refleja un resultado final y pronóstico similar al reportado en la literatura. En este trabajo se observa también a los 10 años una sobrevida de $100 \%$, sin contar con el mismo dato en lo reportado por SEER (37).

La sobrevida global en nuestro estudio se presentó en $85 \%$ a cinco años y en $71 \%$ a 10 años, similar a los resultados del estudio de Migowski en Brasil en 2010, donde obtuvo una sobrevida de 88 y $71 \%$ a cinco años y a 10 años, respectivamente (6).

\section{Limitaciones del estudio}

Es necesario hacer unas precisiones sobre el estudio, dado que se trata de un grupo a quien se le hace seguimiento a partir de un "punto cero", definido como el momento de terminación del tratamiento. Una de las debilidades la puede constituir el hecho de recolectar la información a partir de la historia clínica que en principio no había sido elaborada para tal fin. En este caso se hizo un seguimiento desde la terminación del tratamiento, en forma minuciosa y apoyada en la revisión de todo el proceso de controles clínicos y de laboratorio. 
Otro aspecto por considerar es que no hubo una estandarización de todos los profesionales para el seguimiento de los pacientes, lo que conlleva a falta de homogeneidad en el número y periodicidad de los controles y cualificación de las variables clínicas por tener en cuenta al momento del control.

En un problema de estudio de este tipo, el mejor modelo podría ser un prospectivo de cohorte retrospectiva, tomando como cohorte de estudio al grupo con un tratamiento específico, y otro de control con un medicamento, procedimiento o protocolo determinado.

\section{Agradecimientos}

A todo el personal de Oncólogos del Occidente por su apoyo en la elaboración de la base de datos y el suministro de información para hacer posible la realización de este trabajo.

\section{Declaración de fuentes de financiación y conflictos de interés}

Los autores responsables de la elaboración del presente artículo manifiestan que la financiación en su totalidad para la realización del mismo se dio por medios personales proporcionados por los mismos autores y no se contó con ningún apoyo por parte de entidades públicas o privadas. Además, certifican que no existe ningún conflicto de interés con entidades como Universidades, Instituciones o Empresas aportantes relacionadas al cáncer de próstata y a la realización y publicación del artículo.

\section{Responsabilidad de los autores}

Los autores son los únicos responsables por el contenido y el escrito del trabajo. Todos participaron en la recolección de la información, análisis y redacción del trabajo, y cualquier análisis representa solo el sentir de los autores independiente de las instituciones a las cuales representan.

\section{Referencias}

1. Lopes CO CF. Three-dimensional conformal radiotherapy in prostate cancer patients: rise in interleukin 6 (il-6) but not IL-2, IL-4, IL-5, tumor necrosis factor-alpha, mip-1-alpha, and lif levels. Int J Rad Oncol Bio Phy 2012; 82(4): 1385-1388.

2. Jemal A SR, Ward E, Hao Y, Xu J, Murray T, et al. Cancer statistics 2008. CA: Cancer. J Clin 2008; 58(2): 71-96.

3. Ferlay J PD, Steliarova-Foucher E. Estimates of cancer incidence and mortality in Europe 2008. Eur J Cancer 2010; 46(4): 765-81.

4. Tsikitis VL WB, Guerrero MA. Trends of Incidence and Survival of Gastrointestinal Neuroendocrine Tumors in the United States: A Seer Analysis. J Cancer 2012; 3: 292-302.

5. SEER*Stat software: Surveillance Research Program, National Cancer Institute. In: Surveillance Research Program, NCI; 2011.

6. Migowski A SG. Survival and prognostic factors of patients with clinically localized prostate cancer. RevSaúde Pública 2010; 44(2): 344-52.

7. Organización Panamericana de la Salud. Cáncer en las Américas. Perfiles de país, 2013. Washington, D.C.: 2013.

8. López GG. Registro Poblacional de Cáncer de Manizales-Caldas Quinquenio 2002-2006. 2008.

9. Villegas CR, Chacón JA, Cardona JP, Correa LA. Perfil clínico epidemiológico de los pacientes con cáncer tratados en una Institución de Tercer nivel. Colomb Med 2012; 43(1): 11-18.

10. Gleason DF MG. Prediction of prognosis for prostatic adenocarcinoma by combined histological grading and clinical staging. J Urol 1974; 111(1): 58-64.

11. Stark JR PS, Stampfer MJ, Sinnott JA, Finn S, Eisenstein AS, Ma J, et al. Gleason score and lethal prostate cancer: does $3+4=4+3$ ? J Clin Oncol. 2009; 27(21): 3459-64.

12. NCCN Guidelines Version 3. 2012 prostate cáncer. In; 2012.

13. Brawer MK, Chetner MP, BeatieJ, et al. Screening for prostatic carcinoma with prostate specific antigen. $J$ Urol 1992; 147: 841.

14. Mettlin C, Lee F, Drago J, Murphy GP. The American Cancer Society National Prostate Cancer Detection Project. Findings on the detection of early prostate cancer in 2425 men. Cancer 1991; 67: 2949.

15. Wolf AM, Wender RC, Etzioni RB, et al. American Cancer Society guideline for the early detection of prostate cancer: update 2010. CA Cancer J Clin2010; 60: 70 .

16. Catalona WJ, Richie JP, Ahmann FR, et al. Comparison of digital rectal examination and serum prostate specific antigen in the early detection of prostate cancer: results of a multicenter clinical trial of 6,630 men. J Urol 1994; 151:1283.

17. DeVita Jr VT HS, Rosenburg SA. Chapter 97: Cancer of the Prostate. In: DeVita, Hellman, and Rosenberg's Cancer: Principles \& Practice of Oncology. 9 Ed: LIPPINCOTT; 2009. p. 1221-1269.

18.SEER Cancer Statistics Review, 1973-1999 http://seer.cancer.gov/ csr/1973_1999/ (Accessed on February 25, 2011).

19. Hankey BF, Feuer EJ, CleggLX, et al. Cancer surveillance series: interpreting trends in prostate cancer--part I: Evidence of the effects of screening in recent prostate cancer incidence, mortality, and survival rates. J Natl Cancer Inst 1999; 91:1017.

20. Neppl-Huber C ZM, Coebergh JW, Rapiti E, Rachtan J, Holleczek B, Rosso S, et al. Changes in incidence, survival and mortality of prostate cancer in Europe and the United States in the PSA era: additional diagnoses and avoided deaths. Annals of oncology 2012;23(5): 1325-34.

21. Ries, LAG, Melbert, D, Krapcho, M, et al (Eds). SEER Cancer Statistics Review, 1975-2004, National Cancer Institute, Bethesda, MD 2007. Available at: http:// seer.cancer.gov/csr/1975_2004/ (Accessed on October 16, 2009).

22. Dorr VJ, Williamson SK, Stephens RL. An evaluation of prostate-specific antigen as a screening test for prostate cancer. Arch Intern Med 1993;153: 2529.

23. Sakr WA GD, Crissman JD, et al. High grade prostatic intraepithelial neoplasia (HGPIN) and prostatic adenocarcinoma between the ages of 20-69: an autopsy study of 249 cases. In Vivo 1994; 8(3): 439.

24. Braun DP GD, Staren ED. Predicting survival in prostate cancer: the role of quality of life assessment. Support Care Cancer 2012; 20(6): 1267-74.

25. Rebelo MS RP, Souza MC et al. Sobrevida geral em cinco anos de pacientes com câncer de próstata, assistidos no Instituto Nacional de Câncer / Hospital do CâncerI, no Rio de Janeiro, para o período de 1990 a 1994. In: Instituto Nacional de Cancer Ministerio de Saude; 2002.

26. Bostwick DG GD, Hammond ME, Amin MB, Cohen M, Crawford D, Gospadarowicz M, et al. Prognostic factors in prostate cancer. College of American Pathologists Consensus Statement 1999. Arch Pathol Lab Med2000; 124(7): 995-1000

27. Hebert C. Biochemical Control With Radiotherapy Improves Overall Survival in Intermediate and High-Risk Prostate Cancer Patients Who Have an Estimated 10-Year Overall Survival of $>90 \%$. Int J Radiation Oncol Biol Phys 2012; 83(1): 22-27.

28. Stamey TA MJ, Yemoto CM, Sigal BM, Johnstone IM. Biological determinants of cancer progression in men with prostate cancer. JAMA 1999;281(15): 1395.

29. Martin NE ML, Loda M, Depinho RA. Prognostic determinants in prostate cancer. Can J 2011; 17(6): 429-37.

30. Chan TY PA, Walsh PC, Epstein JI. Prognostic significance of Gleason score $3+4$ versus Gleason score $4+3$ tumor at radical prostatectomy. Urology 2000; 56(5): 823 .

31. Thompson IM AD, Chi C, Goodman PJ, Tangen CM, Lucia MS, Feng Z, et al. Assessing prostate cancer risk: results from the Prostate Cancer Prevention Trial. J Nat Can Inst 2006; 98(8): 529-34.

32. Nam RK TA, Klotz LH, Trachtenberg J, Jewett MA, Appu S, Loblaw DA, Sugar L, et al. Assessing individual risk for prostate cancer. J Clin Oncol. 2007; 25(24): 3582-8.

33. Mosbacher MR SP, Otoole KM, Benson MC, Olsson CA, Brody RA, Ennis RD. Postprostatectomy salvage radiation therapy for prostate cancer: impact of pathological and biochemical variables and prostate fossa biopsy. Can J 2002; 8(3): $242-6$

34. Leborgne F, Aguiar S, Mezerra J, LebogneJH. Irradiación posprostatectomía radical. Confirmación de resultados preliminares previos. Rev Med Urug. 2006; 22(4): 293-8.

35. Kim MM HK, Levy LB, Frank SJ, Pugh TJ, Choi S, Nguyen QN, et al. Improvement in prostate cancer survival over time: a 20-year analysis. Can J 2012; 18(1): $1-8$.

36. Hung AY LL, Kuban DA. Stage T1c Prostate Cancer: A Heterogeneous Category with Widely Varying Prognosis. Can J 2002; 8(6): 440-4.

37. Howlader N, Noone AM, Krapcho M, Neyman N, Aminou R, Altekruse SF, et al (eds). SEER Cancer Statistics Review, 1975-2009 (Vintage 2009 Populations), National Cancer Institute. Bethesda, MD,http://seer.cancer.gov/csr/1975_2009 pops09/, based on November 2011 SEER data submission, posted to the SEER web site, 2012.

38. Chan TY, Partin AW, Walsh PC, Epstein JI. Prognostic significance of Gleason score 3+4 versus Gleason score 4+3 tumor at radical prostatectomy. Urology 2000; 56(5): 823 . 